\title{
Computing Evolutionary Chains in Musical Sequences
}

\author{
Maxime Crochemore \\ Institut Gaspard-Monge \\ Université de Marne-la-Vallée \\ E-mail: mac@univ-mlv.fr
}

\author{
Costas S. Iliopoulos* \\ King's College London \\ Dept. Computer Science \\ csi@dcs.kcl.ac.uk
}

\author{
Yoan J. Pinzon ${ }^{\dagger}$ \\ King's College London \\ Dept. Computer Science \\ pinzon@dcs.kcl.ac.uk
}

Submitted: March 23, 2000; Accepted: May 22, 2000

\begin{abstract}
Musical patterns that recur in approximate, rather than identical, form within the body of a musical work are considered to be of considerable importance in music analysis. Here we consider the "evolutionary chain problem": this is the problem of computing a chain of all "motif" recurrences, each of which is a transformation of ("similar" to) the original motif, but each of which may be progressively further from the original. Here we consider several variants of the evolutionary chain problem and we present efficient algorithms and implementations for solving them.
\end{abstract}

Keywords: String algorithms, approximate string matching, dynamic programming, computerassisted music analysis.

\section{Introduction}

This paper is focused on string-matching problems which arise in computer-assisted music analysis and musical information retrieval. In a recent article ([4]), a number of stringmatching problems as they apply to musical situations were reviewed, and in particular the problem of "Evolution Detection" was introduced and discussed. It was pointed out that no specific algorithms for this problem, either in music or in string-matching in general, exist in the literature. However, it seems that musical patterns, or "motifs" actually 'evolve' in this manner in certain types of composition; an actual case is shown by the successive thematic entries shown in the appended Music Example. A more recent example, from Messiaen's piano work, Vingt Regards sur L'Enfant Jésus, is given in [3].

A musical score can be viewed as a string: at a very rudimentary level, the alphabet (denoted by $\Sigma$ ) could simply be the set of notes in the chromatic or diatonic notation, or

${ }^{*}$ Partially supported by the Royal Society grant CCSLAAR.

${ }^{\dagger}$ Partially supported by an ORS studentship. 
at a more complex level, we could use the GPIR representation of Cambouropoulos [2] as the basis of an alphabet. Although a musical pattern-detection algorithm using approximate matching (allowing the normal edit operations, insertion, deletion and replacement) will detect the occurrence of an evolving pattern in the early stages of its history, once it becomes too different from the original form (past whatever threshold is set by the algorithm or its parameters) it will naturally be rejected. To detect a musical motif which undergoes continuing "evolutionary" change is a more challenging proposition, and is the object of this paper. Musical patterns that recur in approximate, rather than identical, form within a composition (or body of musical work) are considered to be of considerable importance in music analysis. Simple examples are the familiar cases of the standard "tonal" answer in a conventional fugue, or the increasingly elaborated varied reprises of an 18th-century rondo theme; on a more subtle level, the idée fixe in Berlioz's Symphonie Fantastique recurs in a wide variety of different forms throughout the four movements of the symphony. In all these cases, each recurrence can be seen as a transformation of the original motif, and each is roughly equivalently "similar" to the original; a measure of this "similarity" will be preset in an algorithm intended to detect the recurrence of the pattern:

$$
A \cdots A^{\prime} \cdots A^{\prime \prime} \cdots A^{\prime \prime \prime} \cdots
$$

where each of the strings $A^{\prime}, A^{\prime \prime}, A^{\prime \prime \prime}, \ldots$ is similar to $A$ within the maximum edit distance preset in the algorithm.

In this paper we are considering the case where each new recurrence of the pattern is based on the previous one rather than on the original form, somewhat in the manner of a "chain":

$$
A \cdots(A)^{\prime} \cdots\left((A)^{\prime}\right)^{\prime} \cdots\left(\left((A)^{\prime}\right)^{\prime}\right)^{\prime} \cdots
$$

(See Figure 1), where $(X)^{\prime}$ denotes a string similar to a given string $X$ within the maximum edit distance preset in the algorithm. These two types of pattern-repetition may in practice, of course, be indistinguishable in certain circumstances; in case (b), a variant of the pattern may actually cancel out the effect of a previous variant, so the overall distance from the original may remain within the bounds allowed by an algorithm for detecting patterns in case (a).

This class of musical pattern-repetition is not extremely common, but it does exist, as the musical examples given above demonstrate. As well as the obvious musical-analytical interest in detecting such evolutionary pattern-chains, they have importance in any application where they might be missed in detecting approximate repetitions of a pattern (case (a)). These would include automated music-indexing systems for data-retrieval, in which each variant of a motif needs to be detected for efficient indexing; for obvious reasons, it would be desirable for the original pattern, rather than arbitrarily-selected successive variants, to appear as a term in the index table.

Approximate repetitions in musical entities play a crucial role in finding musical similarities amongst different musical entities. The problem of finding a new type of repetitions in a musical score, called evolutionary chains is formally defined as follows: given a string $t$ (the "text") and a pattern $p$ (the "motif"), find whether there exists a sequence 
$u_{1}=p, u_{2}, \ldots, u_{\ell}$ occurring in the text $t$ such that, for all $i \in\{1, \ldots, \ell-1\}, u_{i+1}$ occurs to the right of $u_{i}$ in $t$ and $u_{i}$ and $u_{i+1}$ are "similar" (i.e. they differ by a certain number of symbols).

There was no specific algorithm for the evolution chain problem in the literature. Landau and Vishkin [12] gave an algorithm (LV Algorithm) for the string searching with kdifferences problem: given a text of length $n$ over an alphabet $\Sigma$, an integer $k$ and a pattern of length $m$, find all occurrences of the pattern in the text with at most $k$-differences; the LV algorithm requires $\mathcal{O}\left(n^{2}(\log m+\log |\Sigma|)\right)$ running time. The LV method uses

a complicated data structure (the suffix tree) that makes their algorithm unsuitable for practical use. Furthermore algorithms for exact repetitions are in $[1,6,15]$, approximate repeats treated in $[8,13]$ and quasiperiodicities in $[9,10]$

Here we present an $\mathcal{O}\left(n^{2} m / w\right)$ algorithm for several variants of the problem of computing overlapping evolutionary chains with $k$ differences, where $n$ is the length of the input string, $m$ is the length of the motif and $w$ the length of the computer word. Our methods are practical as well as theoretically optimal. Here we have also studied and implemented the computation of the longest evolutionary chain as well as the chain with least number of errors in total; both algorithms also require $\mathcal{O}\left(n^{2} m / w\right)$ operations.

Several variants to the evolutionary problem are still open. The choice of suitable similarity criteria in music is still under investigation. The use of penalty tables may be more suitable than the $k$-differences criterion in certain applications. Additionally, further investigation whether methods such as [12] can be adapted to solve the above problems is needed.

\section{Basic definitions}

Consider the sequences $t_{1}, t_{2}, \ldots, t_{r}$ and $p_{1}, p_{2}, \ldots, p_{r}$ with $t_{i}, p_{i} \in \Sigma \cup\{\epsilon\}, i \in\{1 \ldots r\}$, where $\Sigma$ is an alphabet, i.e. a set of symbols and $\epsilon$ is the empty string. If $t_{i} \neq p_{i}$, then we say that $t_{i}$ differs to $p_{i}$. We distinguish among the following three types of differences:

1. A symbol of the first sequence corresponds to a different symbol of the second one, then we say that we have a mismatch between the two characters, i.e., $t_{i} \neq p_{i}$.

2. A symbol of the first sequence corresponds to "no symbol" of the second sequence, that is $t_{i} \neq \epsilon$ and $p_{i}=\epsilon$. This type of difference is called a deletion.

3. A symbol of the second sequence corresponds to "no symbol" of the first sequence, that is $t_{i}=\epsilon$ and $p_{i} \neq \epsilon$. This type of difference is called an insertion.

As an example, see Figure 1; in positions 1 and 3 of $t$ and $p$ we have no differences (the symbols "match") but in position 2 we have a mismatch. In position 4 we have a "deletion" and in position 5 we have a "match". In position 6 we have an "insertion", and in positions 7 and 8 we have "matches". Another way of seeing this difference is that one can transform the $t$ sequence to $p$ by performing insertions, deletions and replacements of 


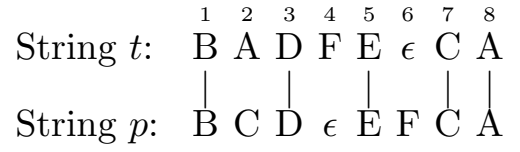

Figure 1: Types of differences: mismatch, deletion, insertion

mismatched symbols. (Without loss of generality, in the sequel we omit the empty string $\epsilon$ from the sequence of symbols in a string).

Let $t=t_{1} t_{2} \ldots t_{n}$ and $p=p_{1} p_{2} \ldots p_{m}$ with $m<n$. We say that $p$ occurs at position $q$ of $t$ with at most $k$-differences (or equivalently, a local alignment of $p$ and $t$ at position $q$ with at most $k$ differences), if $t_{q} \ldots t_{r}$, for some $r>q$, can be transformed into $p$ by performing at most $k$ of the following operations: inserting a symbol, deleting a symbol and replacing a symbol. Furthermore we will use the function $\delta(x, y)$ to denote the minimum number operations (deletions, insertions, replacements) required to transform $x$ into $y$.

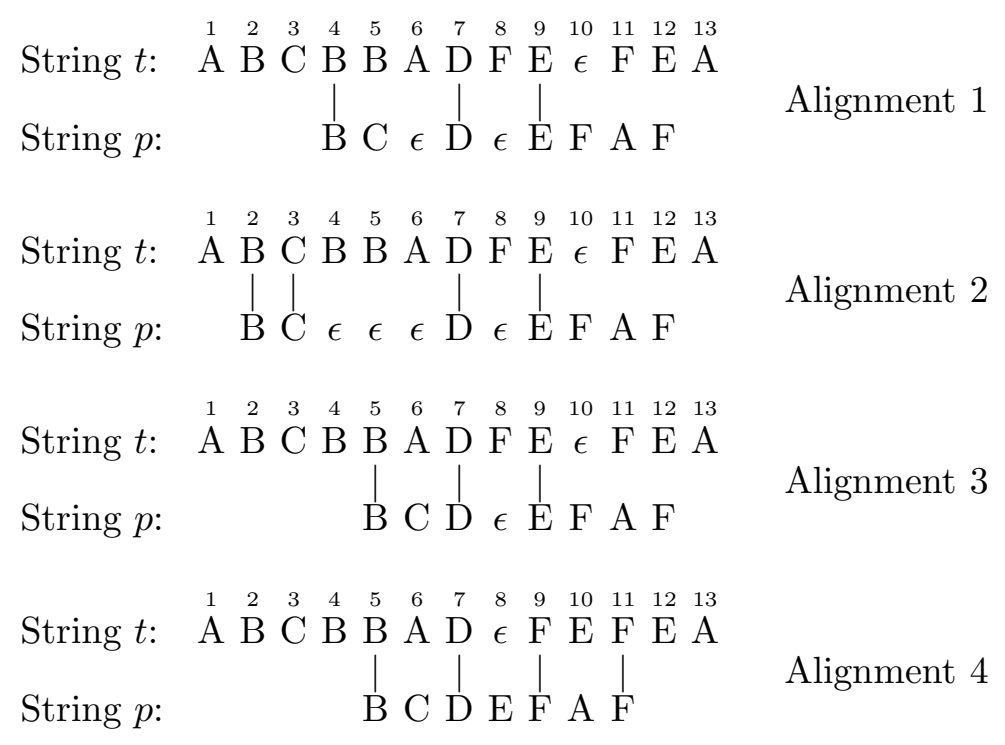

Figure 2: String searching with k-differences.

Let the text $t=A B C B B A D F E F E A$ and the pattern $p=B C D E F A F$ (see Figure 2 ). The pattern $p$ occurs at position 4 of $t$ with at most 6 differences. The pattern $p$ also occurs at position 2 with 7 differences and position 5 with 5 or 4 . The alignment (or alignments) with the minimum number of differences is called an optimal alignment.

In the sequel we also make use of the following graph-theoretic notions: A directed graph $G=(V, E)$ consist of a set $V$ of vertices (nodes) and a set $E$ of edges (arcs). Let $u, v \in V$, then $(u, v)$ denotes the edge between node $u$ and $v$. A path $P$ from $v_{1}$ to $v_{k}$ is a sequence of nodes $\left.P=<v_{1}, v_{2}, \ldots, v_{k}\right\rangle$. $P$ is said to be simple iff the nodes are unique. A cycle in $G$ is a path such that $v_{1}=v_{k}$. A directed acyclic graph $(D A G)$ is a directed graph without cycles. The in-degree $d_{i}^{i n}$ of node $i$ is the number of incoming edges to $i$. 


\begin{tabular}{|c|c|c|c|c|c|}
\hline 0 & 1 & 2 & 3 & 4 & 7 \\
\hline$\epsilon$ & $G$ & $G$ & $\bar{G}$ & $\overline{1}$ & \\
\hline
\end{tabular}

\begin{tabular}{|c|c|c|c|c|c|c|c|c|c|}
\hline 0 & $\epsilon$ & 0 & 0 & 0 & 0 & 0 & 0 & 0 & 0 \\
\hline 1 & $G$ & $\overline{1}$ & 0 & 0 & $\overline{0}$ & 1 & 1 & 1 & 1 \\
\hline 2 & $G$ & $\overline{2}$ & 1 & 0 & $\overline{0}$ & 1 & 2 & $\overline{2}$ & $\overline{2}$ \\
\hline 3 & $G$ & 3 & 2 & 1 & 0 & 1 & 2 & 3 & 3 \\
\hline 4 & $T$ & 3 & 2 & 1 & 1 & 0 & 1 & 2 & 3 \\
\hline 5 & $C$ & 3 & 2 & 2 & 2 & 1 & 0 & 1 & $\overline{2}$ \\
\hline 6 & $\bar{T}$ & 3 & 3 & 3 & 3 & $\overline{2}$ & 1 & $\overline{0}$ & 1 \\
\hline 7 & $\bar{A}$ & 3 & 3 & 3 & 3 & 2 & 2 & 1 & $\overline{0}$ \\
\hline
\end{tabular}

Table 1: The evolutionary matrix $D$ for $t=G G G T C T A$ and $m=3$.

The out-degree $d_{i}^{\text {out }}$ of node $i$ is the number of outgoing edges from $i$. Let $v_{s} \in V$ be the source node and $v_{t} \in V$ be the target node.

Let $c: E \rightarrow \mathbb{Z}$ be a cost function on the edges of $G$. We will also say weight instead of cost. We will write $c(v, u)$ to denote the cost of the edge $(v, u)$. The cost of a path $P=<v_{1}, v_{2}, \ldots, v_{k}>$ is defined to be $c(P)=c\left(v_{1}, v_{2}\right)+\ldots+c\left(v_{k-1}, v_{k}\right)$. The shortest path from a node $v_{s}$ to a node $v_{t}$ is said to be the minimum $c(P)$ over all possible paths from $v_{s}$ to $v_{t}$.

\section{The Evolutionary Matrix}

In this section we present a new efficient algorithm for computing the $n \times n$ evolutionary matrix $D$ : for a given text $t$ of length $n$ and a given integer $m$, we define $D(i, j)$ to be the minimum number of differences between $t_{\max (1, i-m+1)}, \ldots, t_{i}$ and any substring of the text ending at position $j$ of $t$. Informally, the matrix $D$ contains the best scores of the alignments of all substrings of $t$ of length $m$ and any substring of the text. Table 1 shows the evolutionary matrix for $t=G G G T C T A$ and $m=3$.

One can obtain a straightforward $O\left(n^{2} m\right)$ algorithm for computing the evolutionary matrix $D$ by constructing matrices $D^{(s)}[1 . . m, 1 . . n], 1 \leq s \leq n-m$, where $D^{(s)}(i, j)$ is the minimum number of differences between the prefix of the pattern $t_{\max (1, s-m+1)}, \ldots, t_{s}$ and any contiguous substring of the text ending at $t_{j}$; its computation can be based on the Dynamic-Programming procedure presented in [14]. We can obtain $D$ by collating $D^{(1)}$ and the last row of the $D^{(s)}, 2 \leq s \leq n-m$.

Here we will make use of word-level parallelism in order to compute the matrix $D$ more efficiently, similar to the manner used by Myers in [16] and Iliopoulos-Pinzon in [11]. But first we need to compute the $n \times n$ tick-matrix $M$ : if there is an optimal alignment of $t_{\max (1, i-m+1)}, \ldots, t_{i}$ and any contiguous substring of the text ending at $t_{j}$ with the property that there is a difference (i.e. insertion, deletion or mismatch) for $t_{\max (1, i-m+1)}$, then we 


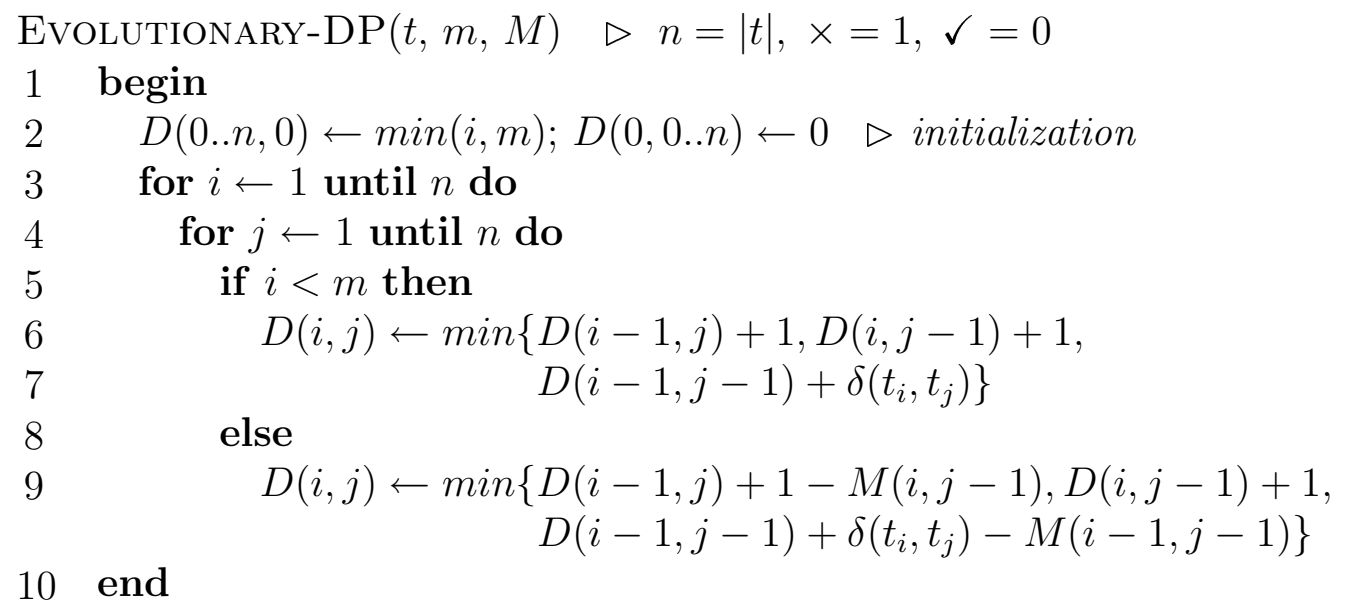

Figure 3: EvOlutionary-DP algorithm

set

$$
M(i, j) \leftarrow \times
$$

otherwise we set

$$
M(i, j) \leftarrow \checkmark
$$

\begin{tabular}{|c|c|c|c|c|c|c|c|c|}
\hline$\epsilon$ & $\times$ & $\times$ & $x$ & $\times$ & $\times$ & $\times$ & $x$ & $\times$ \\
\hline$G$ & $\bar{x}$ & $\checkmark$ & $\bar{\checkmark}$ & $\bar{\checkmark}$ & $x$ & $\times$ & $x$ & $x$ \\
\hline$G$ & $\bar{x}$ & $\bar{x}$ & $\bar{\checkmark}$ & $\bar{\checkmark}$ & $\bar{\checkmark}$ & $\bar{x}$ & $\times$ & $\bar{x}$ \\
\hline$G$ & $x$ & $x$ & $x$ & $\bar{\checkmark}$ & $\bar{\checkmark}$ & $\bar{\checkmark}$ & $x$ & $x$ \\
\hline$T$ & $x$ & $x$ & $\bar{\checkmark}$ & $\bar{\checkmark}$ & $\checkmark$ & $\checkmark$ & $x$ & $\times$ \\
\hline$C$ & $\times$ & $\checkmark$ & $\checkmark$ & $\bar{\checkmark}$ & $\checkmark$ & $\bar{\checkmark}$ & $\checkmark$ & $\times$ \\
\hline$T$ & $\times$ & $\times$ & $x$ & $\times$ & $\times$ & $\checkmark$ & $\checkmark$ & $\checkmark$ \\
\hline$A$ & $\bar{x}$ & $\bar{x}$ & $\bar{x}$ & $\bar{x}$ & $\bar{x}$ & $\bar{x}$ & $\bar{\checkmark}$ & $\bar{\checkmark}$ \\
\hline
\end{tabular}

\begin{tabular}{|c|c|c|c|c|c|c|c|}
0 & 1 & 2 & 3 & 4 & 5 & 6 & 7 \\
\hline$\epsilon$ & $G$ & $G$ & $G$ & $T$ & $C$ & $T$ & $A$ \\
\hline
\end{tabular}

Table 2: The tick-matrix $M$ for $t=G G G T C T A$ and $m=3$.

Assume that the tick-matrix $M[0 . . n, 0 . . n]$ is given. We can use $M$ as an input for the EvolutionARY-DP algorithm (see Fig. 3) to compute the evolutionary matrix $D[0 . . n, 0 . . n]$ as follows:

Theorem 3.1 Given the text $t$, the motif length $m$ and the tick-matrix $M$, the EvOLUTIONARY$\mathrm{DP}$ algorithm correctly computes the matrix $D$ in $\mathcal{O}\left(n^{2}\right)$ units of time. 
Proof. The computation of $D(i, j)$ for all $1 \leq i, j \leq m$, in line 7 , is done using straightforward dynamic programing (see [14]) and thus their values are correct.

Let's consider the computation of $D(i, j)$ for some $i, j>m$. The value of $D(i, j)$ denotes the minimum number of differences in an optimal alignment of a contiguous substring of the text $t_{q} \ldots t_{j}$ (for some $1 \leq q<j$ ) and $t_{i-m+1} \ldots t_{i}$; in that alignment $t_{i}$ can be either to the right of $t_{j}$ or to the left of $t_{j}$ or aligned with $t_{j}$. It is clear that for $q<j:$

$$
D(i, j)=\delta\left(t_{i-m+1} \ldots t_{i}, t_{q} \ldots t_{j}\right)
$$

Now, we will consider all three cases. In the case that the symbol $t_{i}$ is aligned to the right of $t_{j}$, for some $q^{\prime}<j$, we have

$$
\delta\left(t_{i-m+1} \ldots t_{i}, t_{q} \ldots t_{j}\right)=\delta\left(t_{i-m+1} \ldots t_{i-1}, t_{q^{\prime}} \ldots t_{j}\right)+1
$$

Let's consider, for some $\hat{q}<j$

$$
\begin{gathered}
D(i-1, j)=\delta\left(t_{i-m} t_{i-m+1} \ldots t_{i-1}, t_{\hat{q}} \ldots t_{j}\right) \\
\delta\left(t_{i-m} t_{i-m+1} \ldots t_{i-1}, t_{\hat{q}} \ldots t_{j}\right)=\delta\left(t_{i-m}, t_{\hat{q}}\right)+\delta\left(t_{i-m+1} \ldots t_{i-1}, t_{q^{\prime}} \ldots t_{j}\right)
\end{gathered}
$$

Note that

$$
\delta\left(t_{i-m}, t_{\hat{q}}\right)=M(i, j-1)
$$

From equations 1-5 follows that

$$
D(i, j)=D(i-1, j)+1-M(i, j-1)
$$

If $\hat{q} \leq q^{\prime}$, then $t_{i-m}$ is either aligned with $t_{\hat{q}}$ or with $\epsilon$ in an optimal alignment of score $\delta\left(t_{i-m} t_{i-m+1} \ldots t_{i-1}, t_{\hat{q}} \ldots t_{j}\right)$. Thus we have either

$$
\delta\left(t_{i-m} t_{i-m+1} \ldots t_{i-1}, t_{\hat{q}} \ldots t_{j}\right)=\delta\left(t_{i-m}, t_{\hat{q}}\right)+\delta\left(t_{i-m+1} \ldots t_{i-1}, t_{\hat{q}-1} \ldots t_{j}\right)
$$

or

$$
\delta\left(t_{i-m} t_{i-m+1} \ldots t_{i-1}, t_{\hat{q}} \ldots t_{j}\right)=\delta\left(t_{i-m}, \epsilon\right)+\delta\left(t_{i-m+1} \ldots t_{i-1}, t_{\hat{q}} \ldots t_{j}\right)
$$

It is not difficult to see that

$$
\begin{aligned}
\delta\left(t_{i-m+1} \ldots t_{i-1}, t_{q^{\prime}} \ldots t_{j}\right) & =\delta\left(t_{i-m+1} \ldots t_{i-1}, t_{\hat{q}-1} \ldots t_{j}\right) \\
& =\delta\left(t_{i-m+1} \ldots t_{i-1}, t_{\hat{q}} \ldots t_{j}\right)
\end{aligned}
$$

From 1-3, 5, 7 or 8 and 9, we also derive 6 in this subcase.

In the case that the symbol $t_{i}$ is aligned to the left of $t_{j}$ (as above), we have

$$
\delta\left(t_{i-m+1} \ldots t_{i}, t_{q} \ldots t_{j}\right)=\delta\left(t_{i-m+1} \ldots t_{i}, t_{q^{\prime}} \ldots t_{j-1}\right)+1=D(i, j-1)+1
$$

which implies that

$$
D(i, j)=D(i, j-1)+1
$$


In the case that the symbol $t_{i}$ is aligned with $t_{j}$ (as above), we have

$$
\delta\left(t_{i-m+1} \ldots t_{i}, t_{q} \ldots t_{j}\right)=\delta\left(t_{i-m+1} \ldots t_{i-1}, t_{q^{\prime}} \ldots t_{j-1}\right)+\delta\left(t_{i}, t_{j}\right)
$$

In a similar manner as in $2-5$ we can show that

$$
\delta\left(t_{i-m+1} \ldots t_{i-1}, t_{q^{\prime}} \ldots t_{j-1}\right)=D(i-1, j-1)-M(i-1, j-1)
$$

and from 11-12 follows that

$$
D(i, j)=D(i-1, j-1)+\delta\left(t_{i}, t_{j}\right)-M(i-1, j-1)
$$

Equations 6, 10 and 13 show that line 9 of the algorithm correctly compute $D(i, j)$ and the algorithm's correctness follows.

The running time of the EvOLUTIONARY-DP algorithm can easily be shown to be $O\left(n^{2}\right)$.

The key idea behind the computation of $M$ is the use of bit-vector operations that gives us a theoretical speed up factor of $w$ in comparison to the method presented in [14], where $w$ is the compiler word length; thus on a "64-bit computer word" machine one can obtain a speed up of 64 . We maintain the bit-vector

$$
B(i, j)=b_{\ell} \ldots b_{1}
$$

where $b_{r}=1, r \in\{1 \ldots \ell\}, \ell<2 m$, if and only if there is an alignment of a contiguous substring of the text $t_{q} \ldots t_{j}$ (for some $1 \leq q<j$ ) and $t_{i-m+1} \ldots t_{i}$ with $D(i, j$ ) differences such that

- The leftmost $r-1$ pairs of the alignment have $\Sigma_{\ell}^{\ell-r-2} b_{j}$ differences in total.

- the $r$-th pair of the alignment (from left to right) is a difference: a deletion in the pattern, an insertion in the text or a replacement.

Otherwise we set $b_{r}=0$. In other words $B(i, j)$ holds the binary encoding of the path in $D$ to obtain the optimal alignment at $i, j$ with the differences occurring as leftmost as possible.

Given the restraint that the length $m$ of the pattern is less than the length of the computer word, then the "bit-vector" operations allow to update each entry of the matrix $M$ in constant time (using "shift"-type of operation on the bit-vector). The maintenance of the bit-vector is done via operations defined as follows.

- The shift operation moves the bits one position to the left and enter zeros from the right, i.e. $\operatorname{shift}\left(b_{\ell} \ldots b_{1}\right)=b_{\ell-1} \ldots b_{1} 0$

- The shiftc operation shifts and truncates the leftmost bit, i.e. $\operatorname{shift}\left(b_{\ell} \ldots b_{1}\right)=b_{\ell} \ldots b_{1} 0$ 
- Given the integers $x, y, z$, the function $\operatorname{bitmin}(x, y, z)$ returns $r$ one of the integers $\{x, y, z\}$ with the property that $r$ has the least number of 1's (bits set on), and if there is a draw then it returns the one with the leftmost bits (i.e. the maximum of the two when they are viewed as a decimal integer).

- The lastbit operation returns the leftmost bit, i.e. $b_{\ell}$

- The or operation correspond to the bitwise-or operator

The shift, shiftc(x), bitmin, lastbit and or operations can be done in $\mathcal{O}(\mathrm{m} / \mathrm{w})$ time with $\{|x|,|y|,|z|\}<2 m$.

The algorithm in Fig. 4 computes the matrix $M[0 . . n, 0 . . n]$.

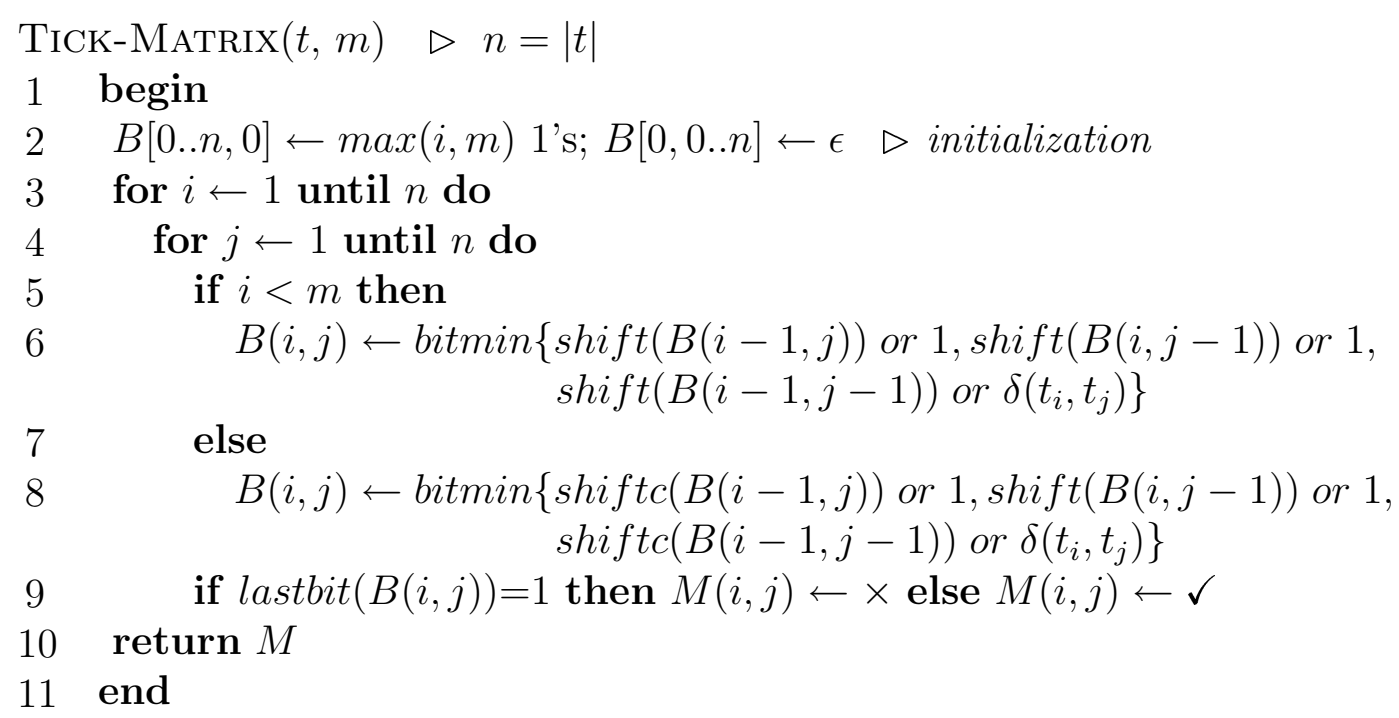

Figure 4: TiCK-MATRIX algorithm

Example. Let the text $t$ be GGGTCT $A$ and $m=3$, the matrix $B$ (Table 3 ) is computed to generate the tick-matrix $M$ (Table 2). Notice that $M(i, j) \leftarrow \times$ if and only if the lastbit $(B(i, j))=1$ and $M(i, j) \leftarrow \checkmark$ otherwise.

Theorem 3.2 The procedure TICK-MATRIX correctly computes the tick-matrix $M$ in $\mathcal{O}\left(n^{2} m / w\right)$ units of time.

Proof. Lines 6 and 8 of the TICK-MATRIX are binary encodings of lines 6 and 8 respectively of the EvOLUTIONARY-DP procedure. The correctness follows from Theorem 1.

Theorem 3.3 The Evolutionary-DP matrix $D$ can be computed in $\mathcal{O}\left(n^{2} m / w\right)$ units of time. 


\begin{tabular}{|c|c|c|c|c|c|c|c|}
\hline 0 & 1 & 2 & 3 & 4 & 5 & 6 & 7 \\
\hline $\bar{\epsilon}$ & $G$ & $G$ & $G$ & $T$ & $C$ & $T$ & $A$ \\
\hline
\end{tabular}

\begin{tabular}{|c|c|c|c|c|c|c|c|c|c|}
\hline & $\bar{\epsilon}$ & & & & & & & & \\
\hline & $G$ & 1 & 0 & 0 & 0 & 1 & 1 & 1 & 1 \\
\hline & $G$ & 11 & 10 & 00 & 00 & 01 & 11 & 11 & 11 \\
\hline & $G$ & 111 & 110 & 100 & 000 & 001 & 011 & 111 & 111 \\
\hline & $T$ & 111 & 101 & 001 & 001 & 000 & 0001 & 110 & $\overline{111}$ \\
\hline & $C$ & 111 & 011 & 011 & 011 & 001 & 000 & $\overline{0001}$ & $\overline{101}$ \\
\hline & $T$ & 111 & 111 & 111 & 111 & 110 & 001 & 000 & 0001 \\
\hline & $A$ & 111 & 111 & 111 & 111 & 101 & 101 & 001 & $\overline{000}$ \\
\hline
\end{tabular}

Table 3: The Bit-Vector Matrix $B$ for $t=G G G T C T A$ and $m=3$.

Proof. The computation of the evolutionary matrix $D$ can be done concurrently with the computation of the matrices $M$ and $B$.

Hence, this algorithm runs in $\mathcal{O}\left(n^{2}\right)$ under the assumption that $m \leq w$, where $w$ is the number of bits in a machine word, i.e., in practical terms the running time is $\mathcal{O}\left(n^{2}\right)$. Also, the space complexity can be reduced to $\mathcal{O}(n)$ by noting that each row of $B, M$ and $D$ depends only on the one immediately preceding row of $B, M$ and $D$ respectively.

\section{Computing the Longest Non-Overlapping Evolu- tionary Chain}

The problem of the longest non-overlapping evolutionary chain (LNOEC) is as follows: given a text $t$ of length $n$, a pattern $p$ of length $m$ and an integer $k<m / 2$, find whether the strings of the sequence $u_{1}=p, u_{2}, \ldots, u_{l}$ occur in $t$ and satisfy the following conditions:

1. $\delta\left(u_{i}, u_{i+1}\right) \leq k$ for all $i \in\{1, \ldots, \ell-1\}$

2. Let $s_{i}$ be the starting position of $u_{i}$ in $t$. Then $s_{i+1}-s_{i} \geq m$ for all $i \in\{1, \ldots, \ell-1\}$

3. Maximizes $\ell$

The method for finding the LNOEC is based on the construction of the evolutionary matrix $D$ presented in the previous section and the graph $G$ defined as follows

Let $G=(V, E)$ be a directed graph where

$$
\begin{gathered}
V=\left\{v_{m}, \ldots v_{n}\right\} \cup\left\{v_{s}, v_{t}\right\} \\
E=\left\{\left(v_{i}, v_{j}\right): D(i, j) \leq k, i \geq m, \quad j-i \geq m\right\} \\
\cup\left\{\left(v_{s}, v_{i}\right): d_{i}^{\text {in }}=0, d_{i}^{\text {out }}>0 \text { for each } v_{i} \in V\right\} \\
\cup\left\{\left(v_{i}, v_{t}\right): d_{i}^{\text {in }}>0, d_{i}^{\text {out }}=0 \text { for each } v_{i} \in V\right\}
\end{gathered}
$$




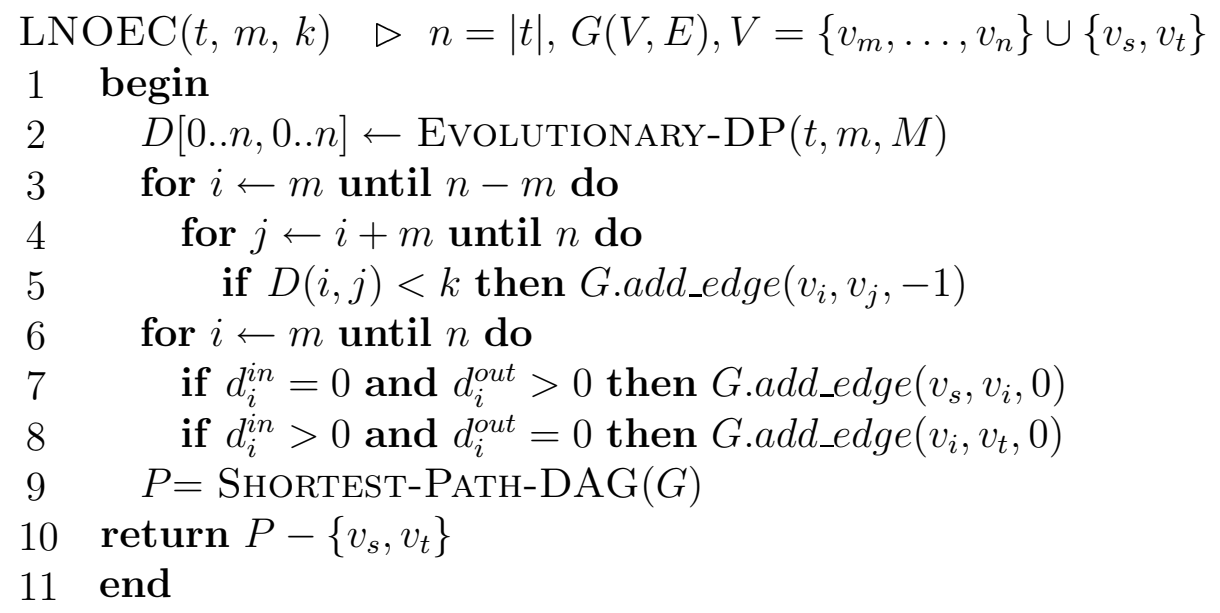

Figure 5: LNOEC algorithm

To complete the construction, we define the cost of an edge as follows

$$
c\left(v_{i}, v_{j}\right)= \begin{cases}0, & \text { if } v_{i}, v_{j} \in\left\{v_{s}, v_{t}\right\} \\ -1, & \text { otherwise }\end{cases}
$$

The problem of finding the LNOEC is equivalent to the problem of finding a shortest (i.e., least-cost) source-to-sink path in $G$. Let us denote the shortest path of $G(s \rightsquigarrow t)$ by $P=<v_{s}, u_{1}, \ldots, u_{\ell}, v_{t}>$. Then we say that the length of the LNOEC is $\ell$.

The time complexity for finding the shortest path of a graph $G$ is known to be $\mathcal{O}(|V| \times$ $|E|)$ in the general case. However, our graph $G$ does not have cycles and all the edges are forward (i.e. for each edge $(u, v) \in V, u$ appears before $v$ ), so $G$ is a topologically sorted DAG. Hence, we can compute the shortest path of $G$ in $\mathcal{O}(|V|+|E|)$ time.

Fig. 8 shows the algorithm to compute the LNOEC. Note that the function G.add_edge $\left(v_{i}, v_{j}, k\right)$ adds the edge $\left(v_{i}, v_{j}\right)$ to the graph $G$, assigning $c\left(v_{i}, v_{j}\right)=k$.

Example. Let the text $t$ be $A B C D A D C B A D, m=3$ and $k=1$. Table 4 shows the evolutionary matrix for the given input. Fig. 6 contains the resulting topologically sorted DAG, the shortest path $P=<v_{s}, 3,7,10, v_{t}>$ (shaded edges), spell out the longest nonoverlapping evolutionary chain, which is $\{A B C, A D C, A D\}$.

\subsection{Running time}

Assuming that $m \leq w$, the time complexity of the algorithm LNOEC is easily seen to be dominated by the complexity of the EvolutionARY-DP algorithm (see Fig. 5 line 2). Hence, the overall complexity for the LNOEC problem will be $\mathcal{O}\left(n^{2}\right)$. Fig. 7 shows the timing $^{1}$ for different values of $m$ and $n$. This algorithm can be generalized for any value

\footnotetext{
${ }^{1}$ Using a SUN Ultra Enterprise $300 \mathrm{MH} z$ running Solaris Unix.
} 


\begin{tabular}{|c|c|c|c|c|c|c|c|c|c|}
\hline & 2 & 3 & 4 & 5 & 6 & 7 & 8 & 9 & 10 \\
\hline$A$ & $B$ & $C$ & $D$ & $A$ & $D$ & $C$ & $B$ & $A$ & $D$ \\
\hline
\end{tabular}

\begin{tabular}{|c|c|c|c|c|c|c|c|c|c|c|c|}
\hline 1 & $A$ & $\overline{0}$ & 1 & 1 & 1 & 0 & 1 & 1 & 1 & 0 & 1 \\
\hline 2 & $B$ & & 0 & 1 & 2 & 1 & 1 & 2 & 1 & 1 & 1 \\
\hline 3 & $C$ & & & 0 & 1 & 2 & 2 & 1 & 2 & 2 & 2 \\
\hline 4 & $D$ & & & & 0 & 1 & 2 & 2 & 2 & 2 & 1 \\
\hline 5 & $A$ & & & & & $\overline{0}$ & 1 & 2 & 2 & 1 & 2 \\
\hline 6 & $D$ & & & & & & 0 & 1 & 2 & 2 & 1 \\
\hline 7 & $C$ & & & & & & & 0 & 1 & 2 & 1 \\
\hline 8 & $B$ & & & & & & & & 0 & 1 & 2 \\
\hline 9 & $A$ & & & & & & & & & 0 & 1 \\
\hline & $D$ & & & & & & & & & & 0 \\
\hline
\end{tabular}

Table 4: The Evolutionary Matrix $D$ for $t=A B C D A D C B A D$ and $m=3$.

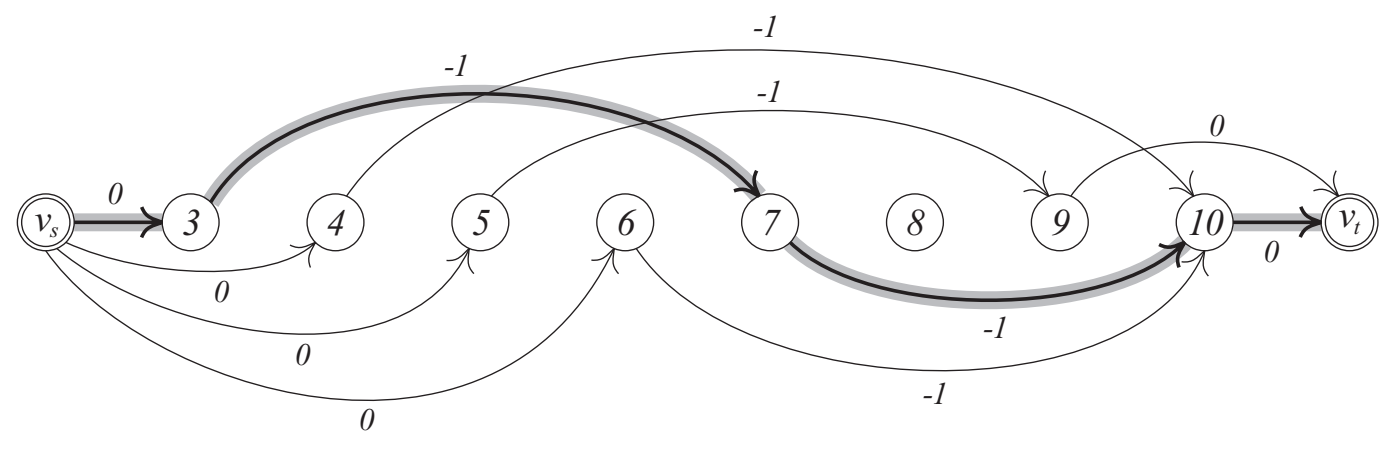

Figure 6: Graph $G$ for $t=A B C D A D C B A D$ and $m=3$.

of $m$ in which case the overall complexity will be $\mathcal{O}\left(n^{2} / m\right)$. The space complexity will be $\mathcal{O}(n+|V|+|E|)$.

\section{Computing the Longest Nearest-Neighbor Non-Overlapping Evolutionary Chain}

The problem of the longest nearest-neighbor non-overlapping evolutionary chain (LNNNOEC) is as follows: given a text $t$ of length $n$, a pattern $p$ of length $m$ and an integer $k<m / 2$, find whether the strings of the sequence $u_{1}=p, u_{2}, \ldots, u_{\ell}$ occur in $t$ and satisfy 


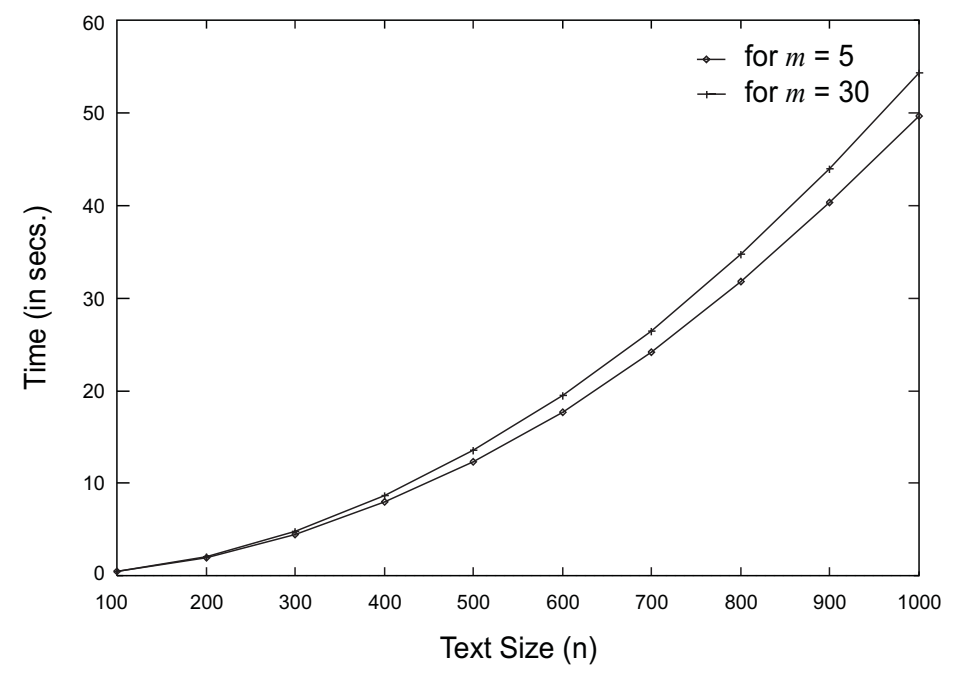

Figure 7: Timing curves for the LNOEC Procedure.

the conditions for LNOEC and minimizes

$$
d=\sum_{i=1}^{\ell-1} \gamma_{i}
$$

where $\gamma_{i}$ is usually the length of the substring (gap) between motif occurrences in the evolutionary chain, i.e. $\gamma_{i}=f\left(s_{i+1}-s_{i}-m\right)$, where $f$ is a penalty table.

The computation of the LNNNOEC can be accomplished by redefining equation 14 as follows

$$
c\left(v_{i}, v_{j}\right)= \begin{cases}0 & , \text { if } v_{i}, v_{j} \in\left\{v_{s}, v_{t}\right\} \\ -n+f\left(s_{i+1}-s_{i}-m\right), & \text { otherwise }\end{cases}
$$

For simplicity, let us assume $f(x)=x$. Fig. 8 shows the algorithm to compute the LNNNOEC.

\section{Computing the Longest Minimum-Weight Non-Overlapping Evolutionary Chain}

The problem of the longest minimum-weight non-overlapping evolutionary chain (LMWNOEC) is as follows: given a text $t$ of length $n$, a pattern $p$ of length $m$ and an integer $k<m / 2$, find whether the strings of the sequence $u_{1}=p, u_{2}, \ldots, u_{\ell}$ occur in $t$ and satisfy the conditions for LNOEC and minimizes

$$
e=\sum_{i=1}^{\ell-1} \delta\left(u_{i}, u_{i+1}\right)
$$




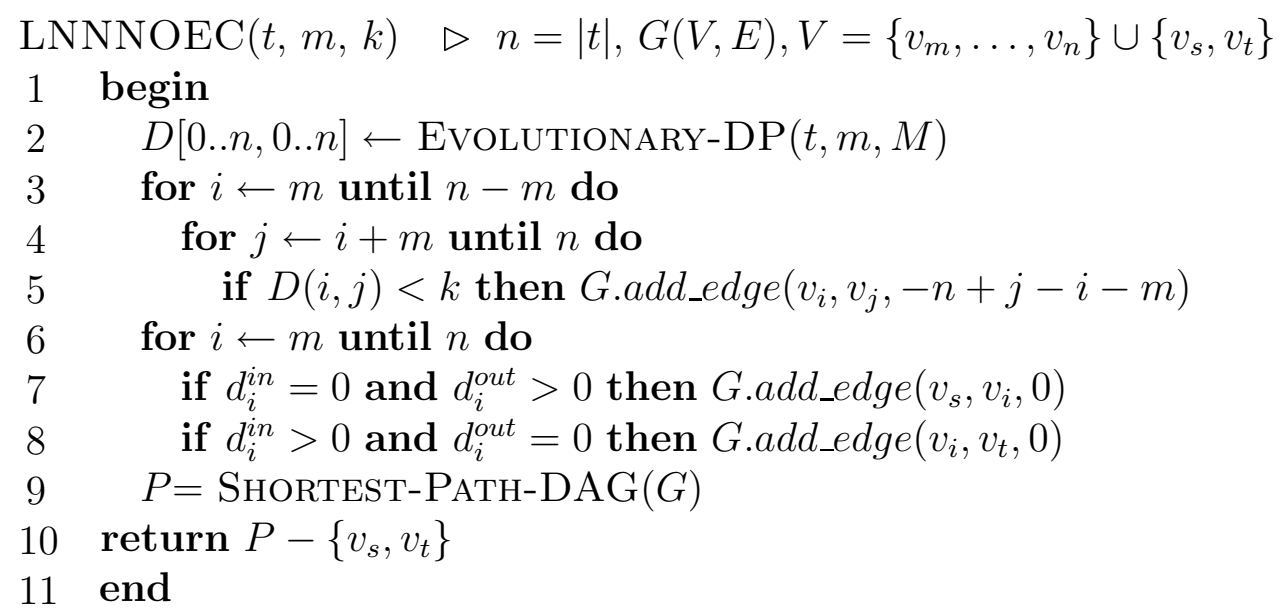

Figure 8: LNNNOEC algorithm

A slightly modification of the LNOEC cost function (Equation 14) will solve the problem

$$
c\left(v_{i}, v_{j}\right)= \begin{cases}0 & , \text { if } v_{i}, v_{j} \in\left\{v_{s}, v_{t}\right\} \\ -n+\delta\left(u_{i}, u_{i+1}\right), & \text { otherwise }\end{cases}
$$

Fig. 9 shows the algorithm for the LMWNOEC problem. 


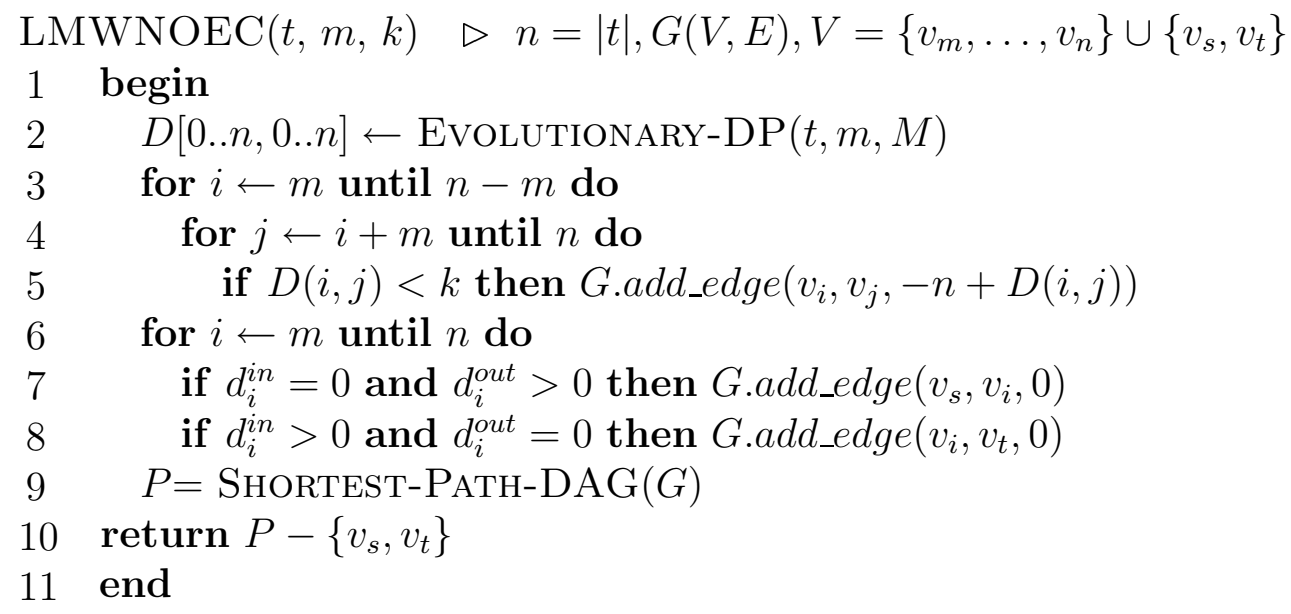

Figure 9: LMWNOEC algorithm

\section{Conclusion and Open problems}

Here we presented practical algorithms for the computation of several variants of the evolutionary chain problem. The the Longest Non-Overlapping Evolutionary Chain, Computing the Longest Nearest-Neighbor Non-Overlapping Evolutionary Chain and Computing the Longest Minimum-Weight Non-Overlapping Evolutionary Chain, which are of practical importance.

The problems presented here need to be further investigated under a variety of similarity or distance rules (see [5]). For example, Hamming distance of two strings $u$ and $v$ is defined to be the number of substitutions necessary to get $u$ from $v$ ( $u$ and $v$ have the same length).

Finally comparisons of the empirical results obtained and to those that can be obtained from software library of string algorithms (see [7]) should be drawn.

\section{References}

[1] A. Apostolico and F. P. Preparata, Optimal Off-line Detection of Repetitions in a String, Theoretical Computer Science, 22 3, pp. 297-315 (1983).

[2] E. Cambouropoulos, A General Pitch Interval Representation: Theory and Applications, Journal of New Music Research 25, pp. 231-251 (1996).

[3] E. Cambouropoulos, T. Crawford and C.S. Iliopoulos, (1999) Pattern Processing in Melodic Sequences: Challenges, Caveats and Prospects. In Proceedings of the AISB'99 Convention (Artificial Intelligence and Simulation of Behaviour), Edinburgh, U.K., pp. 42-47 (1999). 
[4] T. Crawford, C. S. Iliopoulos, R. Raman, String Matching Techniques for Musical Similarity and Melodic Recognition, Computing in Musicology, Vol 11, pp. 73-100 (1998).

[5] T. Crawford, C. S. Iliopoulos, R. Winder, H. Yu, Approximate musical evolution, in the Proceedings of the 1999 Artificial Intelligence and Simulation of Behaviour Symposium (AISB'99), G. Wiggins (ed), The Society for the Study of Artificial Intelligence and Simulation of Behaviour, Edinburgh, pp. 76-81 (1999).

[6] M. Crochemore, An optimal algorithm for computing the repetitions in a word, Information Processing Letters 12, pp. 244-250 (1981).

[7] M. Crochemore, C.S. Iliopoulos and H. Yu, Algorithms for computing evolutionary chains in molecular and musical sequences, Proceedings of the 9-th Australasian Workshop on Combinatorial Algorithms Vol 6, pp. 172-185 (1998).

[8] V. Fischetti, G. Landau, J.Schmidt and P. Sellers, Identifying periodic occurences of a template with applications to protein structure, Proc. 3rd Combinatorial Pattern Matching, Lecture Notes in Computer Science, vol. 644, pp. 111-120 (1992).

[9] C. S. Iliopoulos and L. Mouchard, An $O(n \log n)$ algorithm for computing all maximal quasiperiodicities in strings, Proceedings of CATS'99: "Computing: Australasian Theory Symposium", Auckland, New Zealand, Lecture Notes in Computer Science, Springer Verlag, Vol 21 3, pp. 262-272 (1999).

[10] C. S. Iliopoulos, D. W. G. Moore and K. Park, Covering a string, Algorithmica 16, pp. 288-297 (1996).

[11] C. S. Iliopoulos and Y.J. Pinzon, The Max-Shift Algorithm, submitted.

[12] G. M. Landau and U. Vishkin, Fast parallel and serial approximate string matching, in Journal of Algorithms 10, pp. 157-169 (1989).

[13] G. M. Landau and J. P. Schmidt, An algorithm for approximate tandem repeats, in Proc. Fourth Symposium on Combinatorial Pattern Matching, Springer-Verlag Lecture Notes in Computer Science 648, pp. 120-133 (1993).

[14] G. M. Landau and U. Vishkin, Introducing efficient parallelism into approximate string matching and a new serial algorithm, in Proc. Annual ACM Symposium on Theory of Computing, ACM Press, pp. 220-230 (1986).

[15] G. Main and R. Lorentz, An $O(n \log n)$ algorithm for finding all repetitions in a string, Journal of Algorithms 5, pp. 422-432 (1984).

[16] E. W. Myers, A Fast Bit-Vector Algorithm for Approximate String Matching Based on Dynamic Progamming, in Journal of the ACM 46 3, pp. 395-415 (1999). 